\title{
El valor educativo de la telemedicina
}

\author{
Mila García-Barbero \\ Oficina Europea de la Organización Mundial de la Salud en Barcelona
}

La telemedicina entendida como -el uso de las tecnologías de información y comunicación electrónica para apoyar y facilitar la asistencia sanitaria cuando la distancia separa a los participantes- se ha considerado desde sus comienzos, a principios de los años 60, como un instrumento de mejora en la provisión de servicios sanitarios y, salvo en escasas ocasiones, se ha ignorado su posible papel educativo. En los últimos 10 años se han desarrollado numerosos cursos de formación de pregrado y formación continuada basando en la www como mecanismo de educación a distancia, pero se ha prescindido del potencial de la telemedicina como instrumento de capacitación profesional en todos los niveles de la formación. Los esfuerzos de desarrollo se han enfocado a los registros electrónicos de pacientes, la transmisión de imágenes para diagnóstico, la monitorización de pacientes a larga de distancia y la supervisión de

Correspondencia:

M. García-Barbero

Oficina Europea de la Organización Mundial de la Salud en Barcelona

Marc Aureli 22-26, 08006-Barcelona

mgb@es.euro.who.int

milagbarbero@umh.es actuaciones profesionales o segundas opiniones sin prestar atención al valor educativo que acompaña a todos estos procesos. La telemedicina tiene la ventaja de que aborda los problemas que se le plantean al profesional en la práctica profesional, permite la formación sin necesidad de desplazamientos y no depende de la localización geográfica.

A pesar de su gran potencial tanto clínico como formativo, pocos países han hecho una apuesta política de implantación.

Telemedicine as -the use of electronic information and communication technology to support health care services at a distance1- has been considered since its beginning in the 60s, as a tool to improve health care services and, except in very few situations, its educational potential has been forgotten. In the last 10 years a great number of courses for undergraduate, postgraduate and continuing education of health care professionals have been developed using the www as a distance learning mechanism, but the potential of telemedicine has not been considered for professional development at any level. Development of telemedicine has focus on electronic patients records, patient monitoring at a distance, and supervision of professional capacities or second opinion, not 
considering the educational component of these processes. Telemedicine has the advantage that focus on problems presented to the professionals in their daily practice, allows capacity building without travelling and it does not depend of the geographical location. Despite its great clinical and educative potential, few countries have taken a political decision for its wide implementation.

\section{QUE ENTENDEMOS POR TELEMEDICINA}

Debemos aclarar que la telemedicina entendida como -"el uso de las tecnologías de información y de comunicación electrónicas para apoyar y facilitar la asistencia sanitaria cuando la distancia separa a los participantes" ${ }^{1}$ no abarca todos los posibles instrumentos educativos relacionados con el uso de la informática o de las tecnologías de la comunicación. Cuando hablamos del uso de estas tecnologías debemos diferenciar entre el uso de la informática como plataforma para el aprendizaje y el uso de las tecnologías de la información y comunicación (TIC) como sistema de interacción entre el "profesor" y el "alumno". Términos como ciber-salud o ciber-aprendizaje (eHealth; e.Learning) incluyen cosas tan dispares como el uso de CDs, sistemas de información en ordenadores, bases de datos, registros electrónicos de pacientes, el uso de internet o de e-mail; libros electrónicos, etc. Un CD puede ser un excelente instrumento educativo de formación a distancia pero no entraría dentro del concepto de telemedicina ${ }^{2}$. Las dos palabras claves que definen la telemedicina son comunicación y distancia.

\section{PORQUÉ RESULTA ÚTIL}

Los cambios demográficos de profesionales que dan lugar a la escasez de profesionales cada vez en un mayor número de países ${ }^{3}$, la necesidad de reforzar los mecanismos de seguridad del paciente ${ }^{4}$ el rápido desarrollo del conocimiento en biomedicina y la necesidad de los profesionales de mantener la competencia profesional de por vida, hacen de la telemedicina un instrumento ideal para la formación continuada. A estas situaciones demográficas y geográficas se añade la dificultad de muchos profe- sionales para ausentarse del lugar de trabajo, desplazarse o absorber los costes económicos del aprendizaje presencial. Todo ello convierte a la telemedicina en un medio idóneo para llevar a cabo un aprendizaje pertinente, basado en las necesidades profesionales y con la posibilidad de adaptarse a los horarios mas adecuados, sin necesidad de desplazamiento y a un coste mucho menor 5,6,7,8,10 y 11 . En la formación de pregrado permite el acceso de los estudiantes a un gran número de situaciones clínicas que de otra forma quedarían, en el mejor de los casos, estarían restringidas a un pequeño número.

La pregunta por lo tanto, ya no es si la telemedicina es útil, que está demostrado que lo es, sino que aplicaciones son las más adecuadas para una situación concreta, lo que cambiaría la pregunta a: ¿como la telemedicina, como instrumento de mejora de la asistencia sanitaria, puede apoyar a la vez el desarrollo profesional? y ¿Cómo podemos hacerlo de la manera mas eficaz posible? El aprendizaje con nuevas tecnologías necesita entornos y modelos diferentes a los del aprendizaje tradicional presencial, pero los principios educativos son los mismos y la tecnología solamente un instrumento. El profesor tiene que cambiar de función de "transmisor de información" a asesor y consultor y el alumno pasar de un sujeto pasivo a un aprendiz activo ${ }^{2}$.

\section{CUALES SON LOS MODELOS DE PROVISIÓN DE SERVICIOS ÚTILES PARA LA EDUCACIÓN MÉDICA}

En términos puramente clínicos, la capacidad de actuación de muchos profesionales, tanto de atención primaria como de otras especialidades médicas, se verían altamente reforzada por el apoyo de otros profesionales mediante el uso de aplicaciones de telemedicina. Así mismo, los grandes hospitales de referencia de los que dependen una serie de centros de atención primaria y hospitales comarcales serían más eficientes utilizando sistemas de telemedicina. Se evitarían desplazamientos, esperas y posibilidades de complicaciones en los pacientes y con un buen sistema de transmisión los especialistas podrían saber tanto del paciente como si lo tuvieran delante ${ }^{12}$.

A pesar de las grandes diferencias de infraestructura y por lo tanto de posibilidades de acceso a la información y a otros profesionales entre países y dentro de los países, (figuras 1 y 2 ) y dada la rapi- 
Figura 1. Uso de la tecnología por los médicos de atención primaria en los países de la UE.

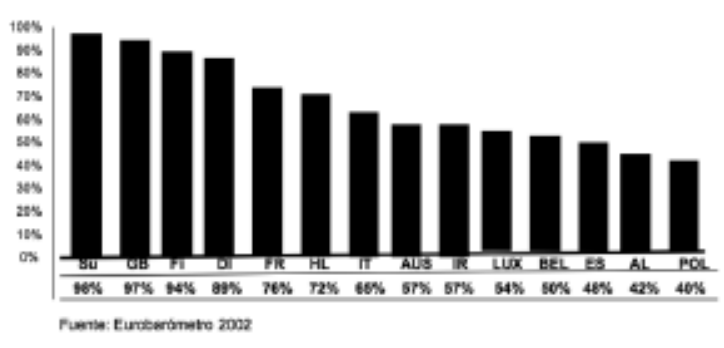

dez de desarrollo de la tecnología, podemos suponer que Internet se convertirá en la plataforma de acceso a la información y formación a global en los próximos 10 años (figura 3). La implantación a nivel global, permitirá el acceso a la su uso no solo en los países más desarrollados, sino que se convertirá en el único medio de información en los países en vías de desarrollo con infraestructuras terrestres altamente deficientes ${ }^{13}$. Esta accesibilidad permitirá que los profesionales consulten con otros profesio- nales los problemas de sus pacientes y mediante la solución continuada de problemas llegar a poder resolverlos ellos mismos.

Basicamente se pueden considerar cuatro modelos diferentes definidos por la forma de uso de la tecnología individual o colectivo y estructurado y ad-hoc y en tiempo real y diferido. Figura 4.

Estas variaciones presentan posibilidades infinitas de aplicación en la gestión de pacientes, la investigación y la educación. Cada una de las modalidades será mas adecuada según los objetivos y las situaciones clínicas, Individual -ad-hoc- tiempo real será el más adecuado en las emergencias clínicas de todo tiempo mientras que Colectivo-programado-tiempo real o diferido será mas adecuado para la formación de grupos específicos.

\section{EL IMPACTO DE LA TELEMEDICINA EN EL DESARROLLO DE PROFESIONALES SANITARIOS}

Todavía existen pocos estudios del impacto de la telemedicina como herramienta de formación pero empieza a ver estudio de los que podemos sacar

Figura 2. Uso de Internet en los médicos de atención primaria en los 15 países de la UE

\begin{tabular}{|c|c|c|c|c|c|}
\hline \multirow[t]{2}{*}{ País } & \multicolumn{5}{|c|}{ Utilización de los médicos de atención primaria } \\
\hline & Ordenadores & PDAs & Internet & $\begin{array}{r}\text { Registros } \\
\text { electrónicos }\end{array}$ & $\begin{array}{r}\text { Centros } \\
\text { con www propia }\end{array}$ \\
\hline Finlandia & 100 & 4 & 100 & 56 & 63 \\
\hline Holanda & 100 & 31 & 100 & 88 & 47 \\
\hline Suecia & 98 & 3 & 93 & 90 & 42 \\
\hline Alemania & 95 & 10 & 57 & 48 & 26 \\
\hline Gran Bretaña & 95 & 18 & 87 & 58 & 27 \\
\hline Francia & 89 & 11 & 80 & 6 & 11 \\
\hline Austria & 82 & 2 & 64 & 55 & 18 \\
\hline Irlanda & 72 & 6 & 18 & 28 & 6 \\
\hline España & 71 & 17 & 43 & 9 & 6 \\
\hline Dinamarca & 70 & 1 & 62 & 62 & 13 \\
\hline Luxemburgo & 68 & 0 & 46 & 30 & 12 \\
\hline Italia & 66 & 0 & 48 & 37 & 6 \\
\hline Bélgica & 66 & 7 & 91 & 42 & 9 \\
\hline Grecia & 52 & 3 & 27 & 17 & 4 \\
\hline Portugal & 37 & 3 & 19 & 3 & 2 \\
\hline Media UE & 77 & 8 & 61 & 42 & 19 \\
\hline
\end{tabular}

Fuente: Eurobarómetro 2001; Taylor y Lexman 2002. en Dubois C-A, McKee,M, Nolte E, editores 2006 
Figura 3. Evolución del uso de Internet según el nivel de desarrollo de los países.

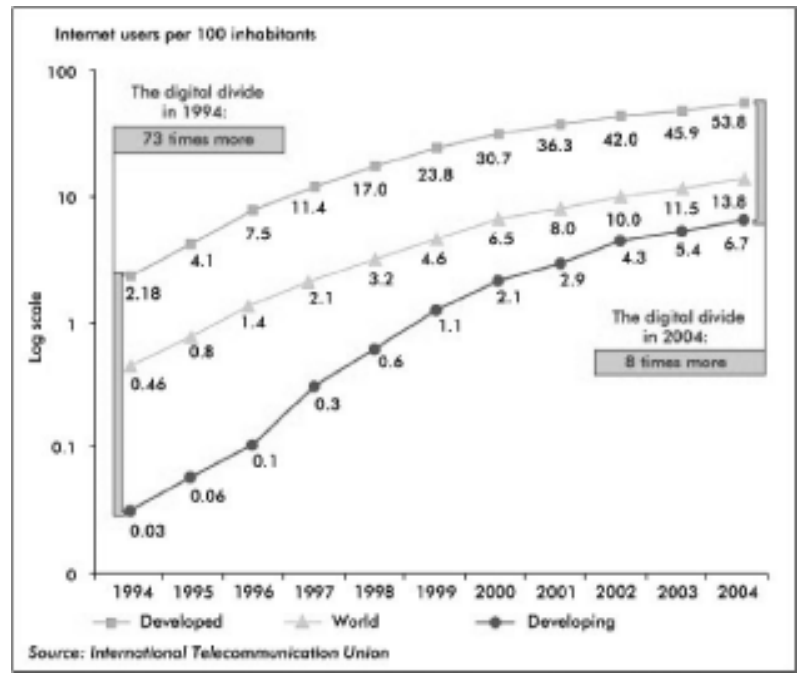

conclusiones indirectas. El programa de dermatología del norte de Noruega en el que médicos generales consultan con los dermatólogos del Hospital Universitario de Trömso los problemas de sus pacientes mediante el uso de una cámara de fotos digital y el correo electrónico, ha conseguido que en 10 años los médicos de primaria sean capaces de solucionar el $90 \%$ de los problemas de dermatología que se les presentan, sin que se haya disminuido la calidad $^{14,15}$. En Viborg County (Dinamarca) el 95\% de las consultas de dermatología ya se solucionaban vía telemedicina en $1999^{16}$. Estos estudios se presentan como avances en el terreno clínico, pero mirándolo desde el punto de vista educativo significa que los médicos de atención primaria han adquirido las competencias necesarias para solucionar la mayoría de los problemas de dermatología de su comunidad.

El proyecto de cooperación internacional Hospital Universitario de Trömso en NoruegaArkhangelsk en el norte de Rusia ha demostrado que el numero de consultas ha ido aumentando con los años que los médicos de Ankladesh han ido abordando mediante telemedicina situaciones mas complejas, siendo capaces de solucionar por si mismos la mayoría de los problemas de las consultas iniciales ${ }^{17}$.

Además de las posibilidades de diagnóstico, la telemedicina ha abierto un amplio campo en lo que se ha denominado cirugía asistida . El ejercito ame- ricano, que fue pionero en el desarrollo de la telemedicina como instrumento de provisión de servicios en sus bases distribuidas en distintos países, ha introducido, programas específicos de formación a distancia adaptados a las necesidades de los profesionales destacados en las distintas partes del mundo $0^{5,18}$. La telemedicina le permite, no solo acceder a distintas bases a la vez, sino adaptar la formación a los problemas y contexto de cada base. Los estudios de calidad de estas intervenciones muestran resultados similares a los obtenidos mediante procedimientos convencionales, aunque con unos periodos de tiempo quirúrgico algo más largos ${ }^{19,20,21}$. No hemos encontrado estudios en los que se haya evaluado el tiempo quirúrgico una vez adquirida la "soltura" necesaria después de la formación, pero

Figura 4. Modelos de uso de la telemedicina como instrumento de formación.

Ejemplo1: Individual -ad-hoc- tiempo real. El equipo de emergencias acude a una llamada de un paciente con dolor torácico y transmite electrocardiagrama al equipo de emergencias del hospital para el correcto diagnostico del paciente.

Ejemplo 2: Individual,-ad-hoc -diferido El Dr Perez tiene un problema con un paciente con una mancha en el antebrazo derecho que no fue capaz de identificar en la primera consulta y ha solicitado ayuda al Dr Gomez dermatólogo de referencia. El Dr Perez envia por e-mail unas fotografias de la mancha del paciente al Dr Gomez que le devuelve al dia siguiente un informe. Si el Dr Gomez examinara la mancha del paciente mediante videoconferencia y con el paciente delante hablaríamos de tiempo real.

Ejemplo 3: Individual,-programado-tiempo real. El Dr García tiene un paciente que requiere una operación urgente que considera no ha realizado suficiente numero de veces para sentirse seguro y no existe posibilidades de traslado del paciente a un centro experimentado. El Dr García realizará la operación bajo la supervisión y consejo del Dr.Hernandez

Ejemplo 4: Individual estructurado. El Dr Perez observa que en su zona hay una alta incidencia de hipotiroidismo con el que no se siente muy familiarizado y solicita ayuda e información del endocrino de referencia . En este programa podrían participar los diversos miembros del mismo equipo de primaria o diversos centros lo que lo convertiría en estructurado y colectivo.

Ejemplo 5: Colectivo-programado-tiempo real. El Dr Gonzalez, cirujano cardiovascular, va a realizar una operación extracorpórea que será retransmitida a una serie de hospitales y presenciada por distintos equipos de cirugía cardiaca.

Ejemplo 6: Colectivo-programado-diferido. El Dr Gonzalez organiza una sesión clínica con varios equipos quirúrgicos en distintos hospitales basándose en la operación de un paciente que realizó la semana pasada. La sesión constará de la proyección de un video de la operación y la discusión de la técnica quirúrgica. 
podemos suponer que se equipara en un corto periodo de tiempo al de los cirujanos cuyo aprendizaje haya sido presencial. La evaluación posterior a la formación utilizando videoconferencias ha mostrado resultados satisfactorio ${ }^{22,23,24}$.

Las especialidades en las que la telemedicina esta mas implantad han sido aquellas en las que existe una necesidad de transmisión de imagen. Radiología, anatomía patológica cirugía, dermatología y cardiología son las especialidades en las que su utilización ha sido a mayor ${ }^{13}$.

En los últimos 5 años están apareciendo centros de formación de profesionales mediante sistemas de telemedicina. Los primeros centros de "teleformación" empezaron hace unos 10 años en Estados Unidos, en Universidades y Centros privados, que vieron en ellos un alto potencial de negocio. En Europa, donde el desarrollo es menor, empieza a haber distintas iniciativas. Holanda quiere extender sus Centros de Formación y tratamiento anticoagulante mediante telemedicina al resto de Europa $^{25}$, el Reino Unido inició en 1999 el programa tele-educación para sus profesionales ${ }^{26}$; HEALTHLINE es un proyecto europeo que ha creado una red de instituciones que comparten, información, programas educativos y consejos a los profesionales o instituciones sanitarias que los requieran ${ }^{27}$. Ciberacademias internacionales y centros de aprendizaje, regional, como la Fundación IAVANTES de Andalucía, el centro de simulación de Israel, tanto a nivel nacional o internacional irán surgiendo cada vez con mas fuerza. Estos centros estarán dotados con las diferentes metodología electrónicas que les permitirán cubrir una amplia gama de necesidades de formación, tanto a tiempo real como diferido.

\section{CONCLUSIONES}

Aunque el uso de la telemedicina está mas extendido como instrumento clínico y de formación entre profesionales sanitarios, tiene un gran potencial como instrumento de formación tanto a nivel de preparado, postgrado o formación continuada. La tecnología no es la panacea a los problemas de la formación de estudiantes o continuada ni genera conocimiento per se, pero sin duda, facilita y apoya su diseminación.

A pesar de la lenta implementación de la telemedicina en los sistemas sanitarios, la necesidad de reducir los costes, asegurar la calidad y facilitar el desarrollo continuado de los profesionales ayudado por la mayor confianza de las nuevas generaciones en el uso de las tecnologías convertirán el uso telemedicina, en un futuro no muy lejano, en un instrumento diario de la práctica médica y de la formación de profesionales. Los sistemas sanitarios tendrán que adaptarse presionados por los ciudadanos y por la competencia de aquellas instituciones mas avanzadas. Así miso las tecnologías de la información y comunicación, ayudaran en cierta medida a solventar el largo debate de la responsabilidad de la formación continuada de los profesionales sanitarios ya que permitirán, a los responsables de la asistencia sanitaria, bien sea el estado, las aseguradoras públicas o las aseguradoras privadas, proporcionar formación relevante a sus profesionales en un gran número de áreas técnicas, a un coste razonable eliminando además los gastos de desplazamiento o sustituciones.

Debido a que el uso de estas tecnologías para la provisión de servicios, puede originar problemas de financiación, éticos y legislativos y requiere un cambio de cultura y organización de los profesionales y las instituciones, su gran potencial de uso está todavía muy lejos de ser alcanzado.

\section{BIBLIOGRAFÍA}

1. Field MJ. Editor; Committee on Evaluating Clinical Applications of Telemedicina., Telemedicine: A Guide to Assessing Telecommunications in Health Care. Institute of Medicine, 1996The National Academies press, Washington DC, USA.

2. García-Barbero, M. La telemedicine como herramienta de formación continuada. Experiencias y perspectivas de futuro. RevistaeSalud 2006, 2 (6) www.revistaesalud.com/revistaesalud/index.php/revistaesalud/article/view/83/176

3. Dubois C-A, McKee,M, Nolte E, editors 2006. JHuman resources for health in Europe. European Observatory on Health Systems and Policies Series. Open University Press Berkshire, Inglaterra

4. World Health Organization, 2005. World Alliance for Patient safety. World health Organization. Geneva, Switzerland.

5. Brumage,M.R.; Chinn,S.; Cho,K., Teleradiology in a military training area; Journal of Telemedicine and Telecare 2001; 7(6):348-9;

6. Crandall,L.A.; Coggan,J.M. Impact of new information technologies on training and continuing education for rural health professionals. Rural Health 1994; 10(3): 208-215;

7. Cornish,P.A.; Church,E.; Callanan,T.; Bethune,C.; Robbins,C.; Miller,R.. Rural interdisciplinary mental health team building via satellite: a demonstration Project. Telemedicine Journal and e-Health 2003;9(1):63-67

8. D'Souza,M.; Hustig,H.; Montgomery,W. Telehealth in rural 
and remote mental health: A review of the use of telepsychiatry in education, training, treatment, discharge planning and a health economic evaluation of the telemedicine service. Australian and New Zealand Journal of Psychiatry $2001 ; 35(4): \mathrm{A} / 7$

9. Haythornthwaite,S. Videoconferencing training for those working with at-risk young people in rural areas of Western Australia. Journal of Telemedicine and Telecare 2002; 8 (suppl3):S3

10. Hussain,P.; Deshpande,A.; Shridhar,P.; Saini,G.; Kay,D. The feasibility of telemedicine for the training and supervision of general practitioners performing ultrasound examinations of patients with urinary tract symptoms. Journal of Telemedicine and Telecare 2004; 10(3):180-2

11. Schopp,L.H.; Johnstone,B.; Reid-Arndt,S. Telehealth brain injury training for rural behavioral health generalists: supporting and enhancing rural service delivery networks. Professional Psychology, Research and Practice 2005; 36(2): 158-63)

12. Smith R. How best to organize acute hospital services?BMJ 2001;323:245-6

13. Misra UK, KalitaJ, Mishra SK, Yadav RK Telemedicina in neurologuy:underutilized potencial. NeurolIndia2005;53:2731 Available from http://www.neurologyindia.con/article.asp? issn $=0028$

14. Josendal O, Fosse G, Andersen KA, Stenvold SE, Falk ES. Distance diagnosis of skin diseases. Tidsskr Nor Laegeforen. 1991;111(1):20-2.

15. Pedersen S; 2005. Telemedicine - a practical experience. Oral presentation. European Forum of Medical Associations and WHO. Oslo, Norway, 11-12 March 2005

16. Klamer, F 1999. Competece in telemedicine. MedCom . Odense

17. Manakova S, 2001. . Report of subsidiary project:
Telemedicine in North-West Russia, 2001. Tromso Telemedicine Center, Tromso, Noruega.

18. Doktor,R.; Johnson,E.; Bangert,D. Designing web based telemedicine training for military health care providers. Journal of Continuing Education in the Health Professions 2001; 21(3):162-69)

19. Eadie,L.H.; Seifalian,A.M.; Davidson,B.R. Telemedicine in surgery [review]. British Journal of Surgery 2003;90 (6) 647-58

20. Burgess,L.P.A. Telemedicine: Teleproctored endoscopic sinus surgery. Laryngoscope 2002;112(2):216-19

21. Link,R.E.; Schulam,P.G.; Kavoussi,L.R. Telesurgery: Remote monitoring and assistance during laparoscopy. Urologic Clinics of North America 2001: 28(1):177-8

22. Brown-Connolly N. Patient satisfaction with telemedical access to specialty services in rural California. Journal of Telemedicine and Telecare 2002;8:7-10

23. Hussain, D. Melville, R. Mannings, D. Curry, D. Kay, and P. Ford. Evaluation of a training and diagnostic ultrasound service for general practitioners using narrowband ISDN [abstract]. Journal of Telemedicine and Telecare 5 (Suppl 1):S1, 1999.

24. Richter, M. Richter, H. U. Wursten, and U. Laffer. Telemedicine: videoconferences in the service of surgery and surgical training? European Surgery - Acta Chirurgica Austriaca 35 (1):55-57, 2003.

25. Hoijtink,E.J. y cols Rascher,I. Telemedicine training and treatment centre "a European rollout of a medical best practice". Studies in Health Technology and Informatics 2005; 114:270-3

26. NHS Executive 1999. -Tele-education http://www.jr2.ox.ac. uk/bandolier/ImpAct/imp02/i2-6.html

27. Arcarese,T.; Boi,S.; Gagliardi,R. Information dissemination and training: Two key issues for consolidating and strengthening the results of health telematic projects. Studies in Health Technology and Informatics 2000;57:137-43 